\section{Subnotificação de acidentes por animais peçonhentos registrados pelo SINAN no Estado do Rio de Janeiro no período de 2001 a 2005*}

\section{Underreporting of accidents with venomous animals registered by SINAN in the State of Rio de Janeiro from 2001 to 2005}

\author{
Judith Tiomny Fiszon ${ }^{1}$ \\ Rosany Bochner² \\ 1 Fundação Oswaldo Cuz - FIOCRUZ; Departamento de Ciências Sociais da \\ Escola Nacional de Saúde Pública Sergio Arouca - ENSP \\ ${ }^{2}$ Fundação Oswaldo Cuz - FIOCRUZ; Instituto de Comunicação e Informação \\ Científica e Tecnológica em Saúde - ICICT; Laboratório de Ciência, Tecnologia e \\ Inovação em Saúde - LabCiTleS
}

* Este estudo foi realizado graças à disponibilização da base de dados da Assessoria de Doenças Imunopreviníveis da Secretaria de Estado de Saúde do Rio de Janeiro, que se encontrava sob a responsabilidade da veterinária Telma Alves Gomes.

Agradecimentos: Agradecemos as contribuições dos revisores que foram muito importantes para a melhoria do trabalho e à veterinária Telma Alves Gomes da SES-RJ.

Correspondência: Judith Tiommy Fiszon. Rua Leopoldo Bulhões, 1480 - sala 909, Rio de Janeiro, RJ. CEP 21041-210. E-mail: jtfiszon@ensp.fiocruz.br

\section{Resumo}

Com o objetivo de verificar se o Sistema de Informação de Agravos de Notificação (SINAN) já permite prescindir de sistemas de informação paralelos voltados ao controle da distribuição do soro, essencial ao atendimento das vítimas de acidentes por animais peçonhentos, foram comparados os dados dos acidentes causados por serpentes, aranhas e escorpiões, disponibilizados pelo SINAN para o Estado do Rio de Janeiro, com os registrados pela Secretaria de Estado de Saúde do Rio de Janeiro (SES-RJ), de 2001 a 2005. Constatou-se o aumento da capacidade do SINAN para registrar os acidentes e a existência de discrepâncias de dados em municípios e regiões do Estado. Foi especialmente alta a subnotificação de acidentes ofídicos pelo SINAN. Discute-se as implicações desse resultado para estudos e pesquisas, bem como para o planejamento e a gestão dos serviços e distribuição dos soros, e concluise que ainda não é possível prescindir dos dados da SES-RJ.

Palavras-chave: Distribuição de soro. Serpentes. Aranhas. Escorpiões.

\footnotetext{
Aprovado pelo Comitê de Ética em Pesquisa em Seres Humanos do Instituto de Pesquisa Clínica Evandro Chagas/FIOCRUZ, Parecer 023/2008
} 


\section{Abstract}

This article has the objective of determining if the Disease Reporting Information System (SINAN) of the State of Rio de Janeiro, Brazil, is adequate enough to do without corresponding information systems directed to serum distribution control, essential to provide care to victims of venomous animals. With this purpose, we compared data of accidents caused by snakes, spiders and scorpions reported to the State Health Department of Rio de Janeiro and those registered in the SINAN from 2001 to 2005. We found an increase in the number of cases reported to SINAN and disagreements in data from municipalities and regions of the State. More specifically, we noticed high underreporting of snake accidents in the SINAN. This paper addresses the implications of these results for studies and research, as well as for planning and management of healthcare services and serum distribution. The conclusion is that it is not possible to rule out data from the Rio de Janeiro State Health Department for the time being.

Keywords: Serum distribution. Snakes. Spiders. Scorpions.

\section{Introdução}

O Sistema de Informação de Agravos de Notificação (SINAN), implantado a partir de 1993, permite acompanhar as doenças de notificação compulsória e quatro outros agravos considerados de interesse nacional: acidentes por animais peçonhentos, atendimento anti-rábico, intoxicações por agrotóxicos e varicela ${ }^{1}$. Desde 1995, a Coordenação Nacional de Controle de Zoonoses e Animais Peçonhentos (CNCZAP) adota o SINAN para consolidar os registros dos acidentes por animais peçonhentos. Esta deliberação gerou uma reação negativa por parte dos municípios e estados, que se mostraram resistentes à adoção do novo sistema, mantendo alguns programas de acompanhamento das informações paralelos e não enviando dados a essa coordenação. Essa reação levou a uma quebra de continuidade nos registros e na perda de qualidade dos dados. Dessa forma, para analisar os acidentes por animais peçonhentos, alguns estudos utilizaram estratégias de análise que combinavam até quatro sistemas nacionais de informação (o SINAN, o SINITOX [Sistema Nacional de Informações Tóxico-Farmacológicas], o SIH-SUS [Sistema de Informações Hospitalares do Sistema Único de Saúde] e o SIM [Sistema de Informações sobre Mortalidade]). Ainda assim, a não interoperabilidade entre esses sistemas implicava em uma análise segmentada, não retratando todos os aspectos relevantes desses acidentes ${ }^{2}$.

Os problemas enfrentados na implantação do SINAN podem ser parcialmente explicados pela baixa valorização dada no passado à informação por parte das instituições e profissionais de saúde ${ }^{3-5}$. No caso dos acidentes por animais peçonhentos, as dificuldades ainda são maiores dado que, historicamente, a informação esteve sempre associada ao controle e distribuição de soro. Todos os esforços voltados a práticas de atendimento e à disponibilização de tratamento específico garantiram o estabelecimento de ações capazes de minimizar a gravidade desses acidentes e, em 
especial, à ampliação da capacidade de produção de soro, que passou a ser suficiente para suprir as necessidades do país. Esses fatores tornaram a manutenção de sistemas de informação atualizados sobre esses acidentes um esforço aparentemente desnecessário, não estimulando as unidades de saúde a alimentar o SINAN com seus dados. Entretanto, é prematuro e carece de bases empíricas supor que acidentes por animais peçonhentos permaneçam estáveis ao longo do tempo, uma vez que as condições ambientais e a ocupação e uso do solo pelo homem estão em constante mudança, fatos que podem gerar um descompasso entre a oferta de serviços de saúde e as várias demandas relacionadas aos acidentes ${ }^{6}$.

Uma limitação importante do SINAN consistia na divulgação restrita de seus dados a uma publicação, o Informe Epidemiológico do SUS, onde disponibilizava apenas os casos distribuídos por agravo e Unidades da Federação. Em 1999, a divulgação dos dados passou a ser feita através do Boletim Eletrônico do SUS, e os acidentes por animais peçonhentos deixaram de ser disponibilizados.

Em julho de 2006 ocorre a disponibilização, na Internet, do banco de dados do SINAN, tornando possível o acesso às informações referentes às doenças e agravos de notificação compulsória ocorridas no país a partir de 2001, bem como o retorno ao acesso às informações acerca dos acidentes por animais peçonhentos ${ }^{7}$. Tal estratégia eleva o SINAN ao mesmo patamar dos demais sistemas nacionais de informação do DATASUS (SIM, SINASC, SIH-SUS, SIA-SUS), que constituem as principais ferramentas dos estudos epidemiológicos, de planejamento e de avaliação em saúde ${ }^{3}$.

Até então, a melhor estratégia para a análise desses acidentes, quando ocorridos no Estado do Rio de Janeiro, era o uso dos registros realizados pela Secretaria de Estado de Saúde do Rio de Janeiro (SESRJ), que tem como base as fichas de notificação dos acidentes. Estes registros cons- tituem um sistema remanescente às práticas de controle e acompanhamento dos acidentes anterior ao SINAN e ainda se mantém como instrumento de controle da distribuição do soro para os municípios no Estado.

A SES-RJ pode não receber as notificações dos casos menos graves em que a aplicação do soro não foi necessária, já que a preocupação com o registro está associada à distribuição e ao controle de estoques de soros específicos e não ao acompanhamento epidemiológico. Os acidentes por animais peçonhentos que implicam na aplicação do soro compreendem todos os casos de ofidismo por serpentes peçonhentas, as formas moderadas e graves de escorpionismo e de araneísmo ${ }^{8-10}$. Acidentes moderados e graves por lagartas do gênero Lonomia sp também devem receber antivenenos, porém não serão objeto do presente estudo ${ }^{9}$. Como conseqüência, parece razoável pressupor que os dados registrados pela SES-RJ apresentem números inferiores aos do SINAN. Uma vez que a maioria dos casos de araneísmo e de escorpionismo são leves ${ }^{8}$, partimos do pressuposto de que as maiores perdas de registros da SES-RJ estariam concentradas nestes, e que as diferenças entre os registros de ofidismo das duas fontes seriam muito pequenas.

O pleno funcionamento do SINAN, explicitado a partir da disponibilização do seu banco de dados através da Internet, sugere que possa ser desnecessária a manutenção de um sistema exclusivo para o controle de distribuição do soro para acidentes por animais peçonhentos. O presente trabalho tem por objetivo verificar se já é possível prescindir destes sistemas paralelos. Para isto, analisa os dados referentes aos acidentes por animais peçonhentos disponibilizados pelo SINAN, comparando-os com os casos registrados pela SES-RJ, com vistas à identificação de possíveis discrepâncias e suas implicações para estudos e pesquisas, bem como para o planejamento e gestão dos serviços e distribuição dos soros. 


\section{Metodologia}

\section{Definição do período de análise}

Uma vez que o SINAN disponibilizou seus dados a partir do ano de 2001, o limite inferior do período não poderia ser anterior a este. E como as duas fontes de informação, SINAN e SES-RJ têm seus dados mais recentes disponibilizados para o ano de 2005, foi selecionado o período de 2001 a 2005 para acompanhar as diferenças entre seus registros.

\section{Descrição dos bancos de dados}

Os dados da SES-RJ referem-se às fichas de notificação de acidentes por animais peçonhentos atendidos no Estado, recebidas pela "Assessoria de Doenças Imunopreviníveis" dessa Secretaria para fins de liberação de soro para os municípios, que são digitadas em um banco de dados próprio. Seu banco de dados contempla tanto o município de atendimento, ou seja, o município que notifica o caso, quanto o município de ocorrência do acidente.

Os dados do SINAN referem-se às fichas de acidentes por animais peçonhentos atendidos e/ou ocorridos no Estado do Rio de Janeiro, cujo fluxo de casos gerado nas unidades de atendimento é enviado para as secretarias municipais de saúde, e destas para as secretarias estaduais de saúde, de onde são encaminhadas ao nível central ${ }^{1}$.

\section{Definição de subnotificação de registros}

A subnotificação anual do SINAN é definida aqui como constituindo a diferença positiva entre a freqüência de acidentes registrada pela SES-RJ e a registrada pelo SINAN em um mesmo ano.

Dada a independência das duas bases, não se pode assegurar que todos os casos registrados pela SES-RJ sejam os mesmos contidos na base do SINAN. Como conseqüência, quando o número de casos registrados pelo SINAN é igual ou superi- or ao da SES-RJ, não faz sentido apresentar o número e o percentual de subnotificação do SINAN.

O percentual de subnotificação anual do SINAN é obtido a partir da seguinte expressão:

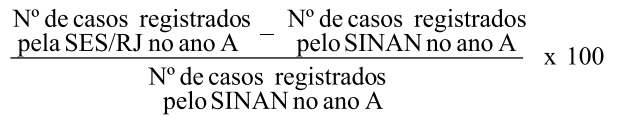

O cálculo da subnotificação do SINAN para o período 2001 a 2005 é obtido pelo somatório das subnotificações anuais.

A subnotificação anual para cada uma das regiões do Estado do Rio de Janeiro é calculada pelo somatório das subnotificações anuais de seus municípios.

\section{Construção de tabelas comparativas}

Para cada um dos animais, serpentes, aranhas e escorpiões, foi construída uma tabela (Tabelas 1, 2 e 3, respectivamente) contendo a freqüência de acidentes por município de notificação e por fonte de registro (SINAN e SES-RJ), para os anos pertencentes ao período de 2001 a 2005. Essas tabelas apresentam também o número de casos subnotificados no período pelo SINAN, para cada município e região. A Tabela 4 apresenta, para cada um dos tipos de acidente, o número e o percentual de subnotificação anual do SINAN.

\section{Resultados}

A Tabela 1 aponta que pelo menos 1.181 casos de acidentes ofídicos não foram notificados ao SINAN no período estudado, sendo as regiões Metropolitana e Serrana responsáveis por $26,4 \%$ e $25,5 \%$ desse total, respectivamente. Os municípios que mais contribuíram para a subnotificação no estado foram Rio de Janeiro, Niterói, Resende e Nova Friburgo, que juntos responderam por uma perda de quase $40 \%$ dos casos do Estado.

A Tabela 2 mostra uma subnotificação de pelo menos 153 casos de acidentes escorpiônicos no período estudado. As regiões 
Tabela 1 - Acidentes ofídicos distribuídos por região, município de notificação e por fonte de registro para os anos de 2001 a 2005 e total de casos subnotificados pelo SINAN no período. Estado do Rio de Janeiro.

Table 1 - Snake accidents distributed per region, reporting city, and record source for the years between 2001 and 2005 and total underreporting by SINAN in the period. State of Rio de Janeiro.

\begin{tabular}{|c|c|c|c|c|c|c|c|c|c|c|c|}
\hline \multirow[b]{2}{*}{ REGIÕES / Municípios } & \multicolumn{2}{|c|}{2001} & \multicolumn{2}{|c|}{2002} & \multicolumn{2}{|c|}{2003} & \multicolumn{2}{|c|}{2004} & \multicolumn{2}{|c|}{2005} & \multirow{2}{*}{$\begin{array}{c}2001-2005 \\
\text { Subnotificações } \\
\text { SINAN }\end{array}$} \\
\hline & SINAN & SES-RJ & SINAN & SES-RJ & SINAN & SES-RJ & SINAN & SES-RJ & SINAN & SES-RJ & \\
\hline METROPOLITANA & 74 & 146 & 65 & 132 & 84 & 141 & 113 & 156 & 99 & 124 & 312 \\
\hline Belford Roxo & - & - & - & - & - & - & - & - & - & - & $*$ \\
\hline Duque de Caxias & 2 & - & 3 & - & 2 & - & 1 & - & - & - & $* * *$ \\
\hline Guapimirim & - & - & - & - & - & - & - & - & - & - & $*$ \\
\hline Itaboraí & 5 & 2 & - & 1 & 2 & 1 & 3 & 3 & 2 & 3 & 2 \\
\hline Itaguaí & - & - & - & - & - & - & - & - & - & - & $*$ \\
\hline Japeri & - & - & - & - & - & - & - & - & - & - & $*$ \\
\hline Magé & 21 & 35 & 11 & 25 & 22 & 22 & 41 & 31 & 18 & 12 & 28 \\
\hline Mangaratiba & 7 & 9 & 13 & 12 & 17 & 18 & 14 & 13 & 10 & 11 & 4 \\
\hline Maricá & - & - & - & - & - & - & - & - & 1 & - & $* * *$ \\
\hline Mesquita & - & - & - & - & - & - & - & - & - & - & * \\
\hline Nilópolis & - & - & - & - & - & - & - & - & - & - & $*$ \\
\hline Niterói & - & 23 & - & 25 & 1 & 23 & 2 & 25 & - & 32 & 125 \\
\hline Nova Iguaçu & 10 & 12 & - & - & - & 2 & - & 4 & - & 10 & 18 \\
\hline Paracambi & 1 & 2 & 4 & 6 & 4 & 2 & 5 & 4 & 5 & 4 & 3 \\
\hline Queimados & - & - & - & - & 2 & - & - & - & - & - & $* * *$ \\
\hline Rio de Janeiro & 28 & 63 & 34 & 63 & 34 & 73 & 47 & 76 & 63 & 52 & 132 \\
\hline São Gonçalo & - & - & - & - & - & - & - & - & - & - & $*$ \\
\hline São João de Meriti & - & - & - & - & - & - & - & - & - & - & $*$ \\
\hline Seropédica & - & - & - & - & - & - & - & - & - & - & * \\
\hline Tanguá & - & - & - & - & - & - & - & - & - & - & * \\
\hline NOROESTE FLUMINENSE & 15 & 36 & 19 & 33 & 16 & 34 & 36 & 46 & 47 & 50 & 91 \\
\hline Aperibé & - & - & - & - & - & - & - & - & - & - & $*$ \\
\hline Bom Jesus do Itabapoana & 2 & 3 & - & - & 5 & 1 & 7 & - & 10 & 13 & 4 \\
\hline Cambuci & - & 2 & - & 1 & - & 2 & - & 6 & - & 2 & 13 \\
\hline Italva & - & - & - & - & - & - & - & - & - & - & $*$ \\
\hline Itaocara & 1 & 1 & 6 & 6 & - & 4 & - & 4 & 6 & 3 & 8 \\
\hline Itaperuna & 1 & 3 & 3 & 4 & 1 & 1 & 5 & 4 & 6 & 3 & 3 \\
\hline Lajé do Muriaé & - & - & 1 & - & - & 1 & - & - & - & - & 1 \\
\hline Miracema & - & 2 & - & 1 & - & 1 & - & 3 & - & 3 & 10 \\
\hline Natividade & 4 & 4 & 5 & 3 & 3 & 8 & 3 & 7 & 2 & 2 & 9 \\
\hline Porciúncula & 7 & 3 & 4 & 5 & 3 & 3 & 6 & 7 & 5 & 5 & 2 \\
\hline Santo Antônio de Pádua & - & - & - & - & - & - & 2 & 2 & 3 & 3 & $* *$ \\
\hline São José de Ubá & - & - & - & - & - & - & - & - & - & - & $*$ \\
\hline Varre-Sai & - & 18 & - & 13 & 4 & 13 & 13 & 13 & 15 & 16 & 41 \\
\hline NORTE FLUMINENSE & 39 & 37 & 19 & 37 & 19 & 26 & 21 & 30 & 37 & 39 & 61 \\
\hline Campos dos Goytacazes & 16 & 14 & 12 & 21 & 10 & 14 & 8 & 7 & 21 & 18 & 13 \\
\hline Carapebus & - & - & - & - & - & - & - & - & - & - & $*$ \\
\hline Cardoso Moreira & - & - & - & - & - & - & - & - & - & - & * \\
\hline Conceição de Macabu & - & - & - & - & - & - & - & - & - & - & * \\
\hline Macaé & - & 10 & - & 6 & - & 5 & - & 15 & 1 & 10 & 45 \\
\hline Quissamã & - & - & - & - & - & - & 1 & - & - & - & $* * *$ \\
\hline São Fidélis & 13 & 13 & 7 & 10 & 9 & 7 & 11 & 8 & 12 & 11 & 3 \\
\hline São Francisco de Itabapoana & 10 & - & - & - & - & - & 1 & - & 3 & - & $* * *$ \\
\hline São João da Barra & - & - & - & - & - & - & - & - & - & - & * \\
\hline SERRANA & 100 & 196 & 109 & 179 & 172 & 207 & 148 & 191 & 185 & 181 & 301 \\
\hline Bom Jardim & 7 & 29 & 5 & 12 & 11 & 21 & - & 20 & 38 & 29 & 59 \\
\hline Cantagalo & - & 14 & 10 & 9 & 16 & 6 & 4 & 3 & 12 & 5 & 14 \\
\hline Carmo & 1 & - & 3 & - & 2 & 3 & 2 & 3 & 1 & 1 & 2 \\
\hline Cordeiro & 5 & 3 & 1 & 1 & 2 & 3 & 2 & 4 & 5 & 6 & 4 \\
\hline Duas Barras & 6 & 8 & 3 & 5 & 13 & 11 & 3 & 5 & 10 & 11 & 7 \\
\hline Macuco & - & - & 1 & - & - & - & - & - & - & - & $* * *$ \\
\hline Nova Friburgo & 7 & 27 & - & 25 & 7 & 29 & 22 & 26 & 24 & 40 & 87 \\
\hline Petrópolis & 28 & 27 & 24 & 27 & 36 & 41 & 34 & 37 & 35 & 36 & 12 \\
\hline Santa Maria Madalena & 5 & 2 & 12 & 11 & 2 & 9 & - & 12 & - & 2 & 21 \\
\hline São José do Vale do Rio Preto & - & 27 & - & 35 & 20 & 21 & 17 & 19 & 15 & 12 & 65 \\
\hline São Sebastião do Alto & - & 1 & 8 & 6 & 5 & 3 & 1 & - & 4 & 5 & 2 \\
\hline Sumidouro & - & 10 & 5 & 9 & 9 & 8 & 17 & 15 & 5 & 4 & 14 \\
\hline Teresópolis & 27 & 35 & 26 & 28 & 34 & 37 & 29 & 30 & 31 & 28 & 14 \\
\hline Trajano de Morais & 14 & 13 & 11 & 11 & 15 & 15 & 17 & 17 & 5 & 2 & $* * *$ \\
\hline
\end{tabular}


Tabela 1 - continuação

Table 1 - continuation

\begin{tabular}{|c|c|c|c|c|c|c|c|c|c|c|c|}
\hline \multirow[b]{2}{*}{ REGIÕES / Municípios } & \multicolumn{2}{|c|}{2001} & \multicolumn{2}{|c|}{2002} & \multicolumn{2}{|c|}{2003} & \multicolumn{2}{|c|}{2004} & \multicolumn{2}{|c|}{2005} & \multirow{2}{*}{$\begin{array}{c}2001-2005 \\
\text { Subnotificações } \\
\text { SINAN }\end{array}$} \\
\hline & SINAN & SES-RJ & SINAN & SES-RJ & SINAN & SES-RJ & SINAN & SES-RJ & SINAN & SES-RJ & \\
\hline BAIXADAS LITORÂNEAS & 11 & 44 & 8 & 41 & 38 & 58 & 41 & 64 & 42 & 52 & 129 \\
\hline Araruama & - & 15 & 6 & 8 & 9 & 8 & 13 & 11 & 13 & 15 & 19 \\
\hline Armação de Búzios & - & - & - & - & 7 & 8 & 8 & 8 & 1 & 1 & 1 \\
\hline Arraial do Cabo & - & - & - & - & - & - & - & - & - & - & * \\
\hline Cabo Frio & 7 & 4 & 2 & 2 & 4 & 8 & 5 & 5 & 11 & 10 & 4 \\
\hline Cachoeiras de Macacu & - & 10 & - & 17 & 4 & 18 & - & 17 & - & 5 & 63 \\
\hline Casimiro de Abreu & 3 & 6 & - & 10 & 9 & 9 & 6 & 7 & 7 & 7 & 14 \\
\hline lguaba Grande & - & - & - & - & - & - & - & - & - & - & $*$ \\
\hline Rio Bonito & 1 & 7 & - & 3 & 4 & 4 & 9 & 12 & 9 & 6 & 12 \\
\hline Rio das Ostras & - & - & - & - & - & - & - & - & - & - & $*$ \\
\hline São Pedro da Aldeia & - & - & - & - & - & - & - & - & 1 & 4 & 3 \\
\hline Saquarema & - & - & - & - & - & - & - & - & - & - & * \\
\hline Silva Jardim & - & 2 & - & 1 & 1 & 3 & - & 4 & - & 4 & 13 \\
\hline MÉDIO PARAÍBA & 57 & 93 & 53 & 103 & 82 & 115 & 64 & 91 & 56 & 73 & 182 \\
\hline Barra do Piraí & - & 3 & 3 & 4 & 3 & 4 & 2 & 8 & 8 & 7 & 11 \\
\hline Barra Mansa & 5 & 11 & 2 & 6 & 16 & 17 & 9 & 11 & 10 & 7 & 13 \\
\hline Itatiaia & - & 5 & 2 & 2 & 2 & 2 & 1 & - & 1 & 2 & 6 \\
\hline Pinheiral & 2 & - & 2 & 2 & 3 & 2 & 2 & 2 & 4 & 1 & $* * *$ \\
\hline Piraí & 7 & 10 & 2 & 15 & 12 & 14 & 3 & 5 & 2 & 6 & 24 \\
\hline Porto Real & - & - & - & - & - & - & 1 & - & 1 & - & $* * *$ \\
\hline Quatis & - & - & 1 & - & 1 & - & - & - & - & - & $* * *$ \\
\hline Resende & 2 & 23 & - & 19 & - & 27 & - & 15 & - & 14 & 96 \\
\hline Rio Claro & 8 & 8 & 12 & 15 & 10 & 13 & 24 & 22 & 9 & 8 & 6 \\
\hline Rio das Flores & - & - & - & - & 1 & 1 & 1 & 2 & 5 & 7 & 3 \\
\hline Valença & 19 & 19 & 12 & 19 & 21 & 20 & 8 & 11 & 11 & 12 & 11 \\
\hline Volta Redonda & 14 & 14 & 17 & 21 & 13 & 15 & 13 & 15 & 5 & 9 & 12 \\
\hline CENTRO-SUL FLUMINENSE & 32 & 57 & 36 & 52 & 54 & 70 & 57 & 61 & 46 & 45 & 84 \\
\hline Areal & - & - & 1 & - & - & - & - & - & - & - & *** \\
\hline Comendador Levy Gasparian & - & - & - & - & - & - & - & - & - & - & * \\
\hline Engenheiro Paulo de Frontin & - & - & - & - & - & - & - & - & - & - & * \\
\hline Mendes & - & - & - & - & - & - & - & - & - & - & * \\
\hline Miguel Pereira & - & 24 & 5 & 13 & 24 & 25 & 28 & 27 & 11 & 10 & 33 \\
\hline Paraíba do Sul & 3 & 9 & - & - & 4 & 6 & 1 & 6 & 5 & 6 & 14 \\
\hline Paty do Alferes & 14 & - & - & - & - & - & - & - & - & - & $* * *$ \\
\hline Sapucaia & 1 & 2 & 3 & 6 & 2 & 3 & - & 1 & 6 & 6 & 6 \\
\hline Três Rios & 10 & 10 & 6 & 10 & 5 & 13 & 4 & 8 & 13 & 14 & 17 \\
\hline Vassouras & 4 & 12 & 21 & 23 & 19 & 23 & 24 & 19 & 11 & 9 & 14 \\
\hline BAÍA DA ILHA GRANDE & 74 & 82 & 59 & 71 & 69 & 63 & 68 & 69 & 72 & 59 & 21 \\
\hline Angra dos Reis & 42 & 48 & 30 & 40 & 42 & 36 & 40 & 41 & 47 & 38 & 17 \\
\hline Parati & 32 & 34 & 29 & 31 & 27 & 27 & 28 & 28 & 25 & 21 & 4 \\
\hline OUTRO ESTADO & 4 & - & 5 & - & 5 & - & 1 & - & - & - & $* * *$ \\
\hline TOTAL ESTADO & 406 & 691 & 373 & 648 & 539 & 714 & 549 & 708 & 584 & 623 & 1181 \\
\hline
\end{tabular}

Fonte: SES-RJ e SINAN

- Dado numérico igual a zero, não resultante de arredondamento; * Não houve notificação de casos pelo SINAN e pela SES-RJ; ** Número de casos notificados pelo SINAN é igual ao número de casos notificados pela SES-RJ; ${ }^{* *}$ Número de casos notificados pelo SINAN é maior que o número de casos notificados pela SES-RJ Source: SES-RJ and SINAN

- Number equal to zero, not a result of rounding; * No cases reported by SINAN or SES-RJ; ** Number of cases reported by SINAN is equal to the number of cases reported by SES-RJ; ***N Number of cases reported by SINAN is greater then the number of cases reported by SES-RJ

Médio Paraíba e Serrana foram responsáveis por $32,0 \%$ e $24,8 \%$ desse total, respectivamente. Os municípios que mais contribuíram para essa subnotificação foram Barra do Piraí, Barra Mansa e Resende, na região Médio Paraíba, e Nova Friburgo, Carmo, Bom Jardim e Cantagalo, na região Serrana, que levaram a uma perda de 75 casos.
Pelo menos 110 casos de acidentes aracnídeos não foram notificados ao SINAN no período estudado de acordo com a Tabela 3. Assim como observado para os acidentes escorpiônicos, as regiões Médio Paraíba e Serrana respondem pelos maiores percentuais de subnotificação, 35,5\% e 33,6\%, respectivamente. Os 
Tabela 2 - Acidentes escorpiônicos distribuídos por região, município de notificação e por fonte de registro para os anos de 2001 a 2005 e total de casos subnotificados pelo SINAN no período. Estado do Rio de Janeiro.

Table 2 - Accidents with scorpions distributed per region, reporting city, and record source for the years between 2001 and 2005 and total underreporting by SINAN in the period. State of Rio de Janeiro.

\begin{tabular}{|c|c|c|c|c|c|c|c|c|c|c|c|}
\hline \multirow[b]{2}{*}{ REGIÕES / Municípios } & \multicolumn{2}{|c|}{2001} & \multicolumn{2}{|c|}{2002} & \multicolumn{2}{|c|}{2003} & \multicolumn{2}{|c|}{2004} & \multicolumn{2}{|c|}{2005} & \multirow{2}{*}{$\begin{array}{c}2001-2005 \\
\text { Subnotificações } \\
\text { SINAN }\end{array}$} \\
\hline & SINAN & SES-RJ & SINAN & SES-RJ & SINAN & SES-RJ & SINAN & SES-RJ & SINAN & SES-RJ & \\
\hline METROPOLITANA & 5 & 3 & 3 & 2 & 9 & 6 & 8 & 6 & 8 & 2 & 4 \\
\hline Belford Roxo & - & - & - & - & - & - & - & - & - & - & $*$ \\
\hline Duque de Caxias & 1 & - & - & - & - & - & - & - & - & - & $* * *$ \\
\hline Guapimirim & - & - & - & - & - & - & - & - & - & - & $*$ \\
\hline Itaboraí & - & - & - & - & - & - & - & - & - & - & * \\
\hline Itaguaí & - & - & - & - & - & - & - & - & - & - & * \\
\hline Japeri & - & - & - & - & - & - & - & - & - & - & * \\
\hline Magé & - & - & - & - & 2 & 2 & - & - & - & - & $* *$ \\
\hline Mangaratiba & 1 & 1 & - & - & - & - & 2 & 2 & - & - & ** \\
\hline Maricá & - & - & - & - & - & - & - & - & - & - & * \\
\hline Mesquita & - & - & - & - & - & - & - & - & - & - & * \\
\hline Nilópolis & - & - & - & - & - & - & - & - & - & - & * \\
\hline Niterói & - & - & - & 1 & - & 1 & - & 1 & - & - & 3 \\
\hline Nova Iguaçu & - & - & - & - & - & - & - & 1 & - & - & 1 \\
\hline Paracambi & - & - & 1 & - & 2 & 1 & 2 & 1 & 3 & 1 & $* * *$ \\
\hline Queimados & - & - & - & - & - & - & - & - & - & - & * \\
\hline Rio de Janeiro & 3 & 2 & 2 & 1 & 5 & 2 & 4 & 1 & 5 & 1 & $* * *$ \\
\hline São Goncalo & - & - & - & - & - & - & - & - & - & - & * \\
\hline São João de Meriti & - & - & - & - & - & - & - & - & - & - & * \\
\hline Seropédica & - & - & - & - & - & - & - & - & - & - & * \\
\hline Tanguá & - & - & - & - & - & - & - & - & - & - & * \\
\hline NOROESTE FLUMINENSE & 11 & 3 & 17 & 20 & 39 & 31 & 20 & 20 & 16 & 17 & 20 \\
\hline Aperibé & - & - & - & - & - & - & - & - & - & - & $*$ \\
\hline Bom Jesus do Itabapoana & - & - & 1 & - & - & - & - & - & - & - & $* * *$ \\
\hline Cambuci & - & - & - & - & - & - & - & - & - & - & * \\
\hline Italva & - & - & - & - & 3 & - & - & - & - & - & $* * *$ \\
\hline Itaocara & - & - & 2 & 3 & 1 & 4 & - & 3 & 2 & 2 & 7 \\
\hline Itaperuna & 5 & - & 8 & 12 & 2 & 4 & 10 & 6 & 8 & 9 & 7 \\
\hline Laje do Muriaé & - & - & - & - & 3 & 2 & - & - & - & - & $* * *$ \\
\hline Miracema & - & 2 & - & - & - & 1 & - & 1 & - & - & 4 \\
\hline Natividade & 4 & - & 1 & - & 13 & 5 & 4 & 4 & 5 & 3 & $* * *$ \\
\hline Porciúncula & 2 & 1 & 5 & 5 & 15 & 13 & 6 & 6 & 1 & 3 & 2 \\
\hline Santo Antônio de Pádua & - & - & - & - & 1 & 1 & - & - & - & - & ** \\
\hline São José de Ubá & - & - & - & - & - & - & - & - & - & - & * \\
\hline Varre-Sai & - & - & - & - & 1 & 1 & - & - & - & - & $* *$ \\
\hline NORTE FLUMINENSE & 35 & 11 & 14 & 10 & 14 & 18 & 33 & 27 & 46 & 18 & 10 \\
\hline Campos dos Goytacazes & 6 & - & 7 & 8 & 5 & 12 & 21 & 21 & 22 & 17 & 8 \\
\hline Carapebus & - & - & - & - & - & - & - & - & - & - & * \\
\hline Cardoso Moreira & - & - & - & - & - & - & - & - & - & - & * \\
\hline Conceição de Macabu & - & - & - & - & - & - & - & - & - & - & * \\
\hline Macaé & - & - & - & - & - & - & - & - & - & - & * \\
\hline Quissamã & - & - & - & - & - & - & - & - & - & - & * \\
\hline São Fidélis & 10 & 11 & 3 & 2 & 7 & 6 & 7 & 6 & - & 1 & 2 \\
\hline São Francisco de Itabapoana & 19 & - & 4 & - & 2 & - & 5 & - & 24 & - & $* * *$ \\
\hline São João da Barra & - & - & - & - & - & - & - & - & - & - & * \\
\hline SERRANA & 4 & 17 & 11 & 17 & 30 & 18 & 40 & 28 & 32 & 19 & 38 \\
\hline Bom Jardim & - & 4 & 1 & 2 & - & 2 & - & - & 1 & 1 & 7 \\
\hline Cantagalo & - & 4 & 4 & 5 & 10 & 2 & 10 & 6 & 8 & 2 & 5 \\
\hline Carmo & 2 & - & - & - & 1 & 8 & 4 & 6 & 2 & - & 9 \\
\hline Cordeiro & - & - & - & - & - & - & - & 2 & - & - & 2 \\
\hline Duas Barras & - & - & - & - & - & - & - & - & 2 & 2 & $* *$ \\
\hline Macuco & - & - & 1 & - & - & - & - & - & - & - & $* * *$ \\
\hline Nova Friburgo & 2 & 8 & - & 7 & 6 & 3 & 12 & 6 & 10 & 10 & 13 \\
\hline Petrópolis & - & - & 2 & - & 9 & 3 & 7 & 3 & 6 & 2 & $* * *$ \\
\hline Santa Maria Madalena & - & - & - & - & 2 & - & - & - & - & - & $* * *$ \\
\hline São José do Vale do Rio Preto & - & - & - & - & - & - & - & - & 1 & 1 & $* *$ \\
\hline São Sebastião do Alto & - & - & - & - & - & - & 1 & 1 & - & - & ** \\
\hline Sumidouro & - & - & - & 1 & 1 & - & - & - & - & - & 1 \\
\hline Teresópolis & - & 1 & 3 & 2 & - & - & 6 & 4 & 2 & 1 & 1 \\
\hline Trajano de Morais & - & - & - & - & 1 & - & - & - & - & - & $* * *$ \\
\hline
\end{tabular}


Tabela 2 - continuação

Table 2 - continuation

\begin{tabular}{|c|c|c|c|c|c|c|c|c|c|c|c|}
\hline \multirow[b]{2}{*}{ REGIÕES / Municípios } & \multicolumn{2}{|c|}{2001} & \multicolumn{2}{|c|}{2002} & \multicolumn{2}{|c|}{2003} & \multicolumn{2}{|c|}{2004} & \multicolumn{2}{|c|}{2005} & \multirow{2}{*}{$\begin{array}{c}2001-2005 \\
\text { Subnotificações } \\
\text { SINAN }\end{array}$} \\
\hline & SINAN & SES-RJ & SINAN & SES-RJ & SINAN & SES-RJ & SINAN & SES-RJ & SINAN & SES-RJ & \\
\hline BAIXADAS LITORÂNEAS & 1 & 1 & 2 & 2 & 3 & 2 & 1 & 1 & 4 & 3 & 3 \\
\hline Araruama & - & - & 1 & 2 & 1 & 1 & - & - & 2 & 1 & 1 \\
\hline Armação de Búzios & - & - & - & - & 1 & - & 1 & 1 & - & 1 & 1 \\
\hline Arraial do Cabo & - & - & - & - & - & - & - & - & 1 & - & $* * *$ \\
\hline Cabo Frio & - & - & 1 & - & 1 & - & - & - & 1 & 1 & $* * *$ \\
\hline Cachoeiras de Macacu & - & - & - & - & - & 1 & - & - & - & - & 1 \\
\hline Casimiro de Abreu & 1 & 1 & - & - & - & - & - & - & - & - & ** \\
\hline Iguaba Grande & - & - & - & - & - & - & - & - & - & - & * \\
\hline Rio Bonito & - & - & - & - & - & - & - & - & - & - & $*$ \\
\hline Rio das Ostras & - & - & - & - & - & - & - & - & - & - & * \\
\hline São Pedro da Aldeia & - & - & - & - & - & - & - & - & - & - & * \\
\hline Saquarema & - & - & - & - & - & - & - & - & - & - & * \\
\hline$\underline{\text { Silva Jardim }}$ & - & - & - & - & - & - & - & - & - & - & * \\
\hline MÉDIO PARAÍBA & 39 & 38 & 61 & 28 & 101 & 65 & 81 & 51 & 127 & 61 & 49 \\
\hline Barra do Piraí & - & 6 & - & 1 & 6 & 5 & 3 & 12 & 14 & 13 & 16 \\
\hline Barra Mansa & 5 & 16 & 3 & 5 & 25 & 24 & 13 & 13 & 24 & 20 & 13 \\
\hline Itatiaia & - & - & 6 & - & 4 & 3 & 7 & - & 4 & 1 & $* * *$ \\
\hline Pinheiral & 20 & 3 & 36 & 10 & 27 & 14 & 33 & 12 & 42 & 6 & $* * *$ \\
\hline Piraí & 1 & 1 & - & 1 & - & - & 2 & - & 1 & - & 1 \\
\hline Porto Real & - & - & - & - & 1 & - & - & - & - & - & $* * *$ \\
\hline Quatis & - & - & - & - & - & - & - & - & - & - & * \\
\hline Resende & - & 2 & - & 2 & - & 5 & - & 2 & - & 1 & 12 \\
\hline Rio Claro & - & - & - & - & - & - & - & - & - & - & * \\
\hline Rio das Flores & 3 & - & 6 & - & 28 & 3 & 12 & - & 26 & - & $* * *$ \\
\hline Valença & 0 & 1 & - & - & 2 & 2 & 1 & 2 & 1 & 3 & 4 \\
\hline Volta Redonda & 10 & 9 & 10 & 9 & 8 & 9 & 10 & 10 & 15 & 17 & 3 \\
\hline CENTRO-SUL FLUMINENSE & 15 & 22 & 21 & 18 & 42 & 18 & 23 & 24 & 35 & 34 & 28 \\
\hline Areal & 4 & - & 2 & - & 2 & - & 1 & - & 3 & - & $* * *$ \\
\hline Comendador Levy Gasparian & - & - & 3 & - & 1 & - & - & - & - & - & $* * *$ \\
\hline Engenheiro Paulo de Frontin & - & - & - & - & - & - & - & - & 1 & - & $* * *$ \\
\hline Mendes & - & - & - & - & - & - & - & - & - & - & * \\
\hline Miguel Pereira & - & - & - & - & - & - & - & - & - & - & * \\
\hline Paraíba do Sul & 1 & 6 & - & - & 34 & 11 & 12 & 15 & 23 & 18 & 8 \\
\hline Paty do Alferes & 1 & - & - & - & - & - & - & - & - & - & $* * *$ \\
\hline Sapucaia & 3 & 9 & 8 & 7 & - & - & 5 & 5 & 4 & 6 & 8 \\
\hline Três Rios & 6 & 6 & 1 & 3 & 3 & 5 & 4 & 4 & 2 & 4 & 6 \\
\hline Vassouras & - & 1 & 7 & 8 & 2 & 2 & 1 & - & 2 & 6 & 6 \\
\hline BAÍA DA ILHA GRANDE & 4 & 4 & 2 & 3 & 4 & 2 & 5 & 5 & 2 & 2 & 1 \\
\hline Angra dos Reis & 1 & 1 & 2 & 3 & 4 & 2 & 4 & 4 & 2 & 2 & 1 \\
\hline Parati & 3 & 3 & - & - & - & - & 1 & 1 & - & - & $* *$ \\
\hline OUTRO ESTADO & - & - & 3 & - & 5 & - & 3 & - & 2 & - & $* * *$ \\
\hline TOTAL ESTADO & 114 & 99 & 134 & 100 & 247 & 160 & 214 & 162 & 272 & 156 & 153 \\
\hline \multirow{2}{*}{\multicolumn{12}{|c|}{$\begin{array}{l}\text { Fonte: SES-RJ e SINAN } \\
\text { - Dado numérico igual a zero, não resultante de arredondamento; }{ }^{*} \text { Não houve notificação de casos pelo SINAN e pela SES-RJj ** Número de casos notificados pelo SINAN } \\
\text { Source: SES-RJ and SINAN }\end{array}$}} \\
\hline & & & & & & & & & & & \\
\hline umber cases renorted & s. & & & & 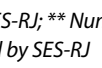 & & & & & & ' \\
\hline
\end{tabular}

municípios da Região Médio Paraíba que mais contribuíram para essa subnotificação foram Barra do Piraí, Resende e Barra Mansa, que levaram a uma perda de 31 casos e na região Serrana foram Nova Friburgo e Bom Jardim, com 30 casos subnotificados.

A Tabela 4 mostra que no Estado do Rio de Janeiro, o SINAN apresentou subnotificação para todos os acidentes em todos os anos do período estudado. Contudo, esse comportamento não é o mesmo para cada uma das regiões do Estado. A subnotificação de ofidismo só não foi observada na região Baía da Ilha Grande nos anos de 2003 e 2005. A subnotificação de escorpionismo ocorreu 
Tabela 3 - Acidentes aracnídeos distribuídos por região, município de notificação e por fonte de registro para os anos de 2001 a 2005 e total de casos subnotificados pelo SINAN no período. Estado do Rio de Janeiro.

Table 3 - Spider accidents distributed per region, reporting city, and record source for the years between 2001 and 2005 and total underreporting by SINAN in the period. State of Rio de Janeiro.

\begin{tabular}{|c|c|c|c|c|c|c|c|c|c|c|c|}
\hline \multirow[b]{2}{*}{ REGIÕES / Municípios } & \multicolumn{2}{|c|}{2001} & \multicolumn{2}{|c|}{2002} & \multicolumn{2}{|c|}{2003} & \multicolumn{2}{|c|}{2004} & \multicolumn{2}{|c|}{2005} & \multirow{2}{*}{$\begin{array}{c}2001-2005 \\
\text { Subnotificações } \\
\text { SINAN }\end{array}$} \\
\hline & SINAN & SES-RJ & SINAN & SES-RJ & SINAN & SES-RJ & SINAN & SES-RJ & SINAN & SES-RJ & \\
\hline METROPOLITANA & 9 & 4 & 8 & 3 & 5 & 5 & 9 & 5 & 11 & 10 & 6 \\
\hline Belford Roxo & - & - & - & - & - & - & - & - & - & - & * \\
\hline Duque de Caxias & - & - & - & - & - & - & - & - & - & - & * \\
\hline Guapimirim & - & - & - & - & - & - & - & - & - & - & * \\
\hline Itaboraí & - & - & - & - & - & - & - & - & - & - & * \\
\hline Itaguaí & - & - & - & - & - & - & - & - & - & - & * \\
\hline Japeri & - & - & - & - & - & - & - & - & - & - & * \\
\hline Magé & - & - & - & - & - & - & - & - & - & - & * \\
\hline Mangaratiba & - & - & - & - & - & - & - & - & - & - & * \\
\hline Maricá & - & - & - & - & - & - & - & - & - & - & * \\
\hline Mesquita & - & - & - & - & - & - & - & - & - & - & * \\
\hline Nilópolis & - & - & - & - & - & - & - & - & - & - & * \\
\hline Niterói & - & 2 & - & 1 & - & 1 & - & - & 2 & 4 & 6 \\
\hline Nova Iguaçu & - & - & - & - & - & - & - & - & - & - & $*$ \\
\hline Paracambi & - & - & - & - & - & - & 1 & - & 1 & - & $* * *$ \\
\hline Queimados & - & - & - & - & - & - & - & - & - & - & $*$ \\
\hline Rio de Janeiro & 7 & 2 & 8 & 2 & 5 & 4 & 7 & 5 & 8 & 6 & $* * *$ \\
\hline São Gonçalo & - & - & - & - & - & - & - & - & - & - & * \\
\hline São João de Meriti & - & - & - & - & - & - & 1 & - & - & - & $* * *$ \\
\hline Seropédica & - & - & - & - & - & - & - & - & - & - & * \\
\hline Tanguá & 2 & - & - & - & - & - & - & - & - & - & $* * *$ \\
\hline NOROESTE FLUMINENSE & 1 & 1 & - & - & 4 & 2 & 1 & 4 & 5 & 4 & 4 \\
\hline Aperibé & - & - & - & - & 1 & - & - & - & - & - & *** \\
\hline Bom Jesus do Itabapoana & - & - & - & - & - & - & - & - & - & - & * \\
\hline Cambuci & - & - & - & - & - & - & - & - & - & - & * \\
\hline Italva & - & - & - & - & - & - & - & - & - & - & * \\
\hline Itaocara & 1 & 1 & - & - & - & - & - & - & - & - & ** \\
\hline Itaperuna & - & - & - & - & 2 & 1 & 1 & 4 & 5 & 4 & 3 \\
\hline Laje do Muriaé & - & - & - & - & - & - & - & - & - & - & * \\
\hline Miracema & - & - & - & - & - & 1 & - & - & - & - & 1 \\
\hline Natividade & - & - & - & - & 1 & - & - & - & - & - & $* * *$ \\
\hline Porciúncula & - & - & - & - & - & - & - & - & - & - & $*$ \\
\hline Santo Antônio de Pádua & - & - & - & - & - & - & - & - & - & - & * \\
\hline São José de Ubá & - & - & - & - & - & - & - & - & - & - & * \\
\hline Varre-Sai & - & - & - & - & - & - & - & - & - & - & $*$ \\
\hline NORTE FLUMINENSE & 2 & 2 & 3 & 2 & - & - & 8 & 10 & 10 & 5 & 2 \\
\hline Campos dos Goytacazes & 1 & 1 & 2 & 2 & - & - & 3 & 4 & 3 & 2 & 1 \\
\hline Carapebus & - & - & - & - & - & - & - & - & - & - & * \\
\hline Cardoso Moreira & - & - & - & - & - & - & - & - & - & - & * \\
\hline Conceição de Macabu & - & - & - & - & - & - & - & - & - & - & * \\
\hline Macaé & - & - & - & - & - & - & - & 1 & 1 & - & 1 \\
\hline Quissamã & - & - & - & - & - & - & - & - & - & - & $*$ \\
\hline São Fidélis & 1 & 1 & - & - & - & - & 5 & 5 & 5 & 3 & $* * *$ \\
\hline São Francisco de Itabapoana & - & - & 1 & - & - & - & - & - & 1 & - & $* * *$ \\
\hline São João da Barra & - & - & - & - & - & - & - & - & - & - & $*$ \\
\hline SERRANA & 8 & 18 & 18 & 26 & 22 & 25 & 70 & 47 & 90 & 40 & 37 \\
\hline Bom Jardim & - & 4 & 5 & 4 & - & 1 & - & 4 & 14 & 9 & 9 \\
\hline Cantagalo & - & - & - & 1 & 2 & - & 12 & 6 & 10 & 3 & 1 \\
\hline Carmo & - & - & - & - & - & - & - & 1 & - & - & 1 \\
\hline Cordeiro & - & - & - & - & - & - & - & 1 & - & - & 1 \\
\hline Duas Barras & - & - & 1 & - & - & - & 1 & 1 & 3 & 1 & $* * *$ \\
\hline Macuco & - & - & - & - & 1 & - & 1 & - & - & - & $* * *$ \\
\hline Nova Friburgo & 5 & 9 & - & 9 & 4 & 12 & 29 & 19 & 43 & 17 & 21 \\
\hline Petrópolis & 1 & 1 & 3 & 3 & 5 & 3 & 16 & 5 & 8 & 3 & $* * *$ \\
\hline Santa Maria Madalena & - & - & - & - & - & - & - & - & - & - & $*$ \\
\hline São José do Vale do Rio Preto & - & 1 & - & - & - & - & 1 & 1 & 4 & 3 & 1 \\
\hline São Sebastião do Alto & - & - & - & - & - & - & - & - & - & - & * \\
\hline Sumidouro & - & - & - & 1 & - & - & 3 & 1 & 3 & 1 & 1 \\
\hline Teresópolis & 2 & 3 & 9 & 8 & 10 & 9 & 7 & 8 & 5 & 3 & 2 \\
\hline Trajano de Morais & - & - & - & - & - & - & - & - & - & - & $*$ \\
\hline
\end{tabular}


Tabela 3 - continuação

Table 3 - continuation

\begin{tabular}{|c|c|c|c|c|c|c|c|c|c|c|c|}
\hline \multirow[b]{2}{*}{ REGIÕES / Municípios } & \multicolumn{2}{|c|}{2001} & \multicolumn{2}{|c|}{2002} & \multicolumn{2}{|c|}{2003} & \multicolumn{2}{|c|}{2004} & \multicolumn{2}{|c|}{2005} & \multirow{2}{*}{$\begin{array}{c}2001-2005 \\
\text { Subnotificações } \\
\text { SINAN }\end{array}$} \\
\hline & SINAN & SES-RJ & SINAN & SES-RJ & SINAN & SES-RJ & SINAN & SES-RJ & SINAN & SES-RJ & \\
\hline BAIXADAS LITORÂNEAS & - & 1 & 2 & 3 & 3 & 2 & 7 & 4 & 6 & 5 & 5 \\
\hline Araruama & - & 1 & 2 & 2 & 2 & 2 & 2 & 3 & 4 & 3 & 2 \\
\hline Armação de Búzios & - & - & - & - & 1 & - & 3 & - & 1 & 1 & *** \\
\hline Arraial do Cabo & - & - & - & - & - & - & - & - & - & - & $*$ \\
\hline Cabo Frio & - & - & - & 1 & - & - & - & - & - & 1 & 2 \\
\hline Cachoeiras de Macacu & - & - & - & - & - & - & - & 1 & - & - & 1 \\
\hline Casimiro de Abreu & - & - & - & - & - & - & - & - & - & - & * \\
\hline Iguaba Grande & - & - & - & - & - & - & - & - & - & - & $*$ \\
\hline Rio Bonito & - & - & - & - & - & - & 2 & - & - & - & $* * *$ \\
\hline Rio das Ostras & - & - & - & - & - & - & - & - & 1 & - & $* * *$ \\
\hline São Pedro da Aldeia & - & - & - & - & - & - & - & - & - & - & $*$ \\
\hline Saquarema & - & - & - & - & - & - & - & - & - & - & * \\
\hline Silva Jardim & - & - & - & - & - & - & - & - & - & - & $*$ \\
\hline MÉDIO PARAÍBA & 12 & 17 & 22 & 14 & 31 & 27 & 30 & 27 & 52 & 26 & 39 \\
\hline Barra do Piraí & - & 3 & 2 & 5 & 2 & 5 & 4 & 9 & 6 & 5 & 14 \\
\hline Barra Mansa & - & 4 & 2 & 2 & 4 & 5 & 2 & 2 & 8 & 5 & 5 \\
\hline Itatiaia & - & 2 & 6 & 1 & 6 & 4 & 4 & - & 7 & 2 & 2 \\
\hline Pinheiral & 2 & - & 4 & - & 3 & 1 & 6 & 1 & 7 & 2 & $* * *$ \\
\hline Piraí & - & - & 1 & 2 & 5 & 1 & 1 & - & 3 & - & 1 \\
\hline Porto Real & 1 & - & - & - & - & - & 1 & - & - & - & $* * *$ \\
\hline Quatis & - & - & 2 & - & - & - & - & - & 1 & - & $* * *$ \\
\hline Resende & - & 1 & - & - & - & 3 & - & 7 & 3 & 4 & 12 \\
\hline Rio Claro & 2 & - & 1 & - & - & - & 4 & 2 & 1 & - & $* * *$ \\
\hline Rio das Flores & 1 & - & - & - & 2 & - & 1 & - & 10 & - & *** \\
\hline Valença & 3 & 4 & 3 & 2 & 4 & 3 & 1 & 1 & 2 & 1 & 1 \\
\hline Volta Redonda & 3 & 3 & 1 & 2 & 5 & 5 & 6 & 5 & 4 & 7 & 4 \\
\hline CENTRO-SUL FLUMINENSE & 4 & 6 & 1 & 1 & 5 & 3 & 12 & 14 & 15 & 18 & 11 \\
\hline Areal & - & - & 1 & - & - & - & - & - & - & - & $* * *$ \\
\hline Comendador Levy Gasparian & - & - & - & - & - & - & 2 & - & - & - & $* * *$ \\
\hline Engenheiro Paulo de Frontin & - & - & - & - & - & - & - & - & - & - & $*$ \\
\hline Mendes & - & - & - & - & - & - & - & - & - & - & * \\
\hline Miguel Pereira & - & - & - & - & - & - & - & 1 & - & - & 1 \\
\hline Paraíba do Sul & 2 & 4 & - & - & 3 & 1 & 4 & 4 & 9 & 11 & 4 \\
\hline Paty do Alferes & - & - & - & - & 1 & - & - & - & - & 1 & 1 \\
\hline Sapucaia & - & - & - & - & - & - & - & - & - & - & $*$ \\
\hline Três Rios & - & - & - & - & - & 1 & 3 & 4 & 4 & 4 & 2 \\
\hline Vassouras & 2 & 2 & - & 1 & 1 & 1 & 3 & 5 & 2 & 2 & 3 \\
\hline BAÍA DA ILHA GRANDE & 10 & 10 & 4 & 9 & 3 & 3 & 7 & 7 & 13 & 3 & 6 \\
\hline Angra dos Reis & 3 & 4 & 2 & 6 & 2 & 2 & 3 & 3 & 8 & 2 & 5 \\
\hline Parati & 7 & 6 & 2 & 3 & 1 & 1 & 4 & 4 & 5 & 1 & 1 \\
\hline OUTRO ESTADO & - & - & 3 & - & - & - & 1 & - & 5 & - & $* * *$ \\
\hline TOTAL ESTADO & 46 & 59 & 61 & 58 & 73 & 67 & 145 & 118 & 207 & 111 & 110 \\
\hline
\end{tabular}

Fonte: SES-RJ e SINAN

- Dado numérico igual a zero, não resultante de arredondamento; ${ }^{*}$ Não houve notificação de casos pelo SINAN e pela SES-RJ; ** Número de casos notificados pelo SINAN é igual ao número de casos notificados pela SES-RJ; *** Número de casos notificados pelo SINAN é maior que o número de casos notificados pela SES-RJ Source: SES-RJ and SINAN

- Number equal to zero, not a result of rounding; * No cases reported by SINAN or SES-RJ; ** Number of cases reported by SINAN is equal to the number of cases reported by SES-RJ;

***Number of cases reported by SINAN is greater then the number of cases reported by SES- $R J$

em todos os anos para as regiões Noroeste Fluminense, Médio Paraíba e Centro-Sul Fluminense, sendo que essas duas últimas regiões foram as únicas que apresentaram subnotificação de araneísmo em todos os anos do período estudado.

\section{Discussão}

A comparação entre os dados consolidados, provenientes das duas fontes de informação, permite afirmar que vem havendo uma crescente captação de notifi- 
Tabela 4 - Números e percentuais de subnotificação dos casos registrados pelo SINAN em relação aos casos registrados pela SES-RJ, com base no SINAN, distribuídos por animal agressor e ano para cada região. Estado do Rio de Janeiro, 2001 a 2005.

Table 4 - Numbers and percentages of underreporting of cases registered by SINAN in relation to the cases registered by SES$R J$, based on SINAN, distributed by type of animal and year for each region, State of Rio de Janeiro, 2001 to 2005.

\begin{tabular}{|c|c|c|c|c|c|}
\hline REGIÕES & $\begin{array}{c}2001 \\
\text { № }(\%)\end{array}$ & $\begin{array}{c}2002 \\
\mathrm{~N}^{\circ}(\%)\end{array}$ & $\begin{array}{c}2003 \\
\text { № (\%) }\end{array}$ & $\begin{array}{c}2004 \\
\text { № }(\%)\end{array}$ & $\begin{array}{c}2005 \\
\mathrm{~N}^{\circ}(\%)\end{array}$ \\
\hline \multicolumn{6}{|c|}{ METROPOLITANA } \\
\hline Serpente & $77(104,1)$ & $71(109,2)$ & $64(76,2)$ & $56(49,6)$ & $44(44,4)$ \\
\hline Escorpião & $* * *$ & $1(33,3)$ & $1(11,1)$ & $2(22,2)$ & $* * *$ \\
\hline Aranha & $2(22,2)$ & $1(12,5)$ & $1(20,0)$ & $* * *$ & $2(18,2)$ \\
\hline \multicolumn{6}{|c|}{ NOROESTE FLUMINENSE } \\
\hline Serpente & $25(166,7)$ & $17(89,5)$ & $22(137,5)$ & $18(50,0)$ & $9(19,1)$ \\
\hline Escorpião & $2(18,2)$ & $5(29,4)$ & $6(15,4)$ & $4(20,0)$ & $3(18,8)$ \\
\hline Aranha & $* *$ & * & $1(25,0)$ & $3(300,0)$ & $* * *$ \\
\hline \multicolumn{6}{|c|}{ NORTE FLUMINENSE } \\
\hline Serpente & $10(25,6)$ & $18(94,7)$ & $9(47,4)$ & $15(71,4)$ & $9(24,3)$ \\
\hline Escorpião & $1(2,9)$ & $1(7,1)$ & $7(50,0)$ & $* * *$ & $1(2,2)$ \\
\hline Aranha & $* *$ & $* * *$ & * & $2(25,0)$ & $* * *$ \\
\hline \multicolumn{6}{|l|}{ SERRANA } \\
\hline Serpente & $104(104,0)$ & $78(71,6)$ & $50(29,1)$ & $47(31,8)$ & $22(11,9)$ \\
\hline Escorpião & $15(375,0)$ & $10(90,9)$ & $9(30,0)$ & $4(10,0)$ & $* * *$ \\
\hline Aranha & $10(125,0)$ & $11(61,1)$ & $9(40,9)$ & $7(10,0)$ & $* * *$ \\
\hline \multicolumn{6}{|c|}{ BAIXADAS LITORÂNEAS } \\
\hline Serpente & $36(327,3)$ & $33(412,5)$ & $21(55,3)$ & $25(61,0)$ & $14(33,3)$ \\
\hline Escorpião & $* *$ & $1(50,0)$ & $1(33,3)$ & ** & $1(25,0)$ \\
\hline Aranha & $1(x)$ & $1(50,0)$ & $* * *$ & $2(28,6)$ & $1(16,7)$ \\
\hline \multicolumn{6}{|c|}{ MÉDIO PARAÍBA } \\
\hline Serpente & $38(66,7)$ & $51(96,2)$ & $36(43,9)$ & $31(48,4)$ & $26(46,4)$ \\
\hline Escorpião & $20(51,3)$ & $6(9,8)$ & $6(5,9)$ & $12(14,8)$ & $5(3,9)$ \\
\hline Aranha & $11(91,7)$ & $5(22,7)$ & $7(22,6)$ & $12(40,0)$ & $4(7,7)$ \\
\hline \multicolumn{6}{|c|}{ CENTRO-SUL FLUMINENSE } \\
\hline Serpente & $39(121,9)$ & $17(47,2)$ & $16(29,6)$ & $10(17,5)$ & $2(4,34)$ \\
\hline Escorpião & $12(80,0)$ & $3(14,3)$ & $2(4,8)$ & $3(13,0)$ & $8(22,9)$ \\
\hline Aranha & $2(50,0)$ & $1(100,0)$ & $1(20,0)$ & $4(33,3)$ & $3(20,0)$ \\
\hline \multicolumn{6}{|c|}{ BAÍA DA ILHA GRANDE } \\
\hline Serpente & $8(10,8)$ & $12(20,3)$ & $* * *$ & $1(1,5)$ & $* * *$ \\
\hline Escorpião & $* *$ & $1(50,0)$ & $* * *$ & $* *$ & $* *$ \\
\hline Aranha & $1(10,0)$ & $5(125,0)$ & $* *$ & $* *$ & $* * *$ \\
\hline \multicolumn{6}{|c|}{ TOTAL ESTADO } \\
\hline Serpente & $337(83,0)$ & $297(79,6)$ & $218(40,4))$ & $203(37,0)$ & $126(21,6)$ \\
\hline Escorpião & $50(43,9)$ & $28(20,9)$ & $32(13,0)$ & $25(11,6)$ & $18(6,6)$ \\
\hline Aranha & $27(58,7)$ & $24(39,34)$ & $19(26,0)$ & $30(20,7)$ & $10(4,8)$ \\
\hline
\end{tabular}

Fonte: SES-RJ e SINAN

(x) Número de casos notificados pelo SINAN é igual a zero; * Não houve notificação de casos pelo SINAN e pela SES-RJ; ** Número de casos notificados pelo SINAN é igual ao número de casos notificados pela SES-RJ; ${ }^{* *}$ Número de casos notificados pelo SINAN é maior que o número de casos notificados pela SES-RJ Source: SES-RJ and SINAN

(x) Number of cases reported by SINAN is equal to zero; * No cases reported by SINAN or SES-RJ; ** Number of cases reported by SINAN is equal to the number of cases reported by SES-RJ; *** Number of cases reported by SINAN is greater then the number of cases reported by SES-RJ 
cações pelo SINAN entre 2001 e 2005. Este aumento da capacidade de captar os casos de acidentes por animais peçonhentos não impede, no entanto, a permanência de grandes diferenças entre os números de casos registrados, tanto nos municípios quanto nas regiões para os três tipos de acidentes, fato que impõe cuidados na análise de seus dados por parte dos pesquisadores, bem como esforços dos gestores para uma maior adesão ao sistema nacional de informação.

Merecem especial atenção os municípios de Niterói, Nova Iguaçu, Cambuci, Miracema, Santa Maria Madalena, Cachoeiras de Macacu, Silva Jardim e Resende, que, apesar de possuírem pólos de atendimento de acidentes por animais peçonhentos $^{7}$, não notificaram acidentes ofídicos ao SINAN em 2005, e enviaram 72 notificações à SES-RJ neste ano. Estes municípios mostram uma clara preocupação com a obtenção de soro, cujo controle ainda está vinculado à apresentação da ficha de notificação do acidente à SES-RJ. Este comportamento nos faz supor que não haja clareza da importância de se ter um sistema de informações unificado, capaz de subsidiar desde análises epidemiológicas até vir a se constituir num instrumento útil para o controle e a distribuição do soro de maneira mais eficiente do que o atualmente estabelecido. Esta compreensão é fundamental para a incorporação da atividade de alimentação do sistema de informações do SINAN à rotina dos serviços locais de saúde.

Como conseqüência das perdas de casos de acidentes ofídicos pelo SINAN, os dados da SES-RJ ainda são extremamente relevantes. Não obstante, dadas às diferenças regionais e municipais, devemos observar as duas bases de dados para deliberar ações por parte da gestão da atenção aos acidentes para evitar problemas por falta de provisão de estoques de soro, que pode vir a ser uma conseqüência direta da observação exclusiva de uma delas. Em alguns municípios, o SINAN já constitui uma base importante para a vigilância desse agravo. Contudo, em 2005 houve ainda por parte do SINAN uma subnotificação de 126 casos de acidentes ofídicos. Tal subnotificação pode resultar, na melhor das hipóteses, em um déficit de 504 ampolas de soro somente nesse ano, isso se considerarmos apenas acidentes botrópicos leves, cujas vítimas devem receber no mínimo de duas a quatro ampolas de soro ${ }^{8,9}$.

No período estudado foram identificados 153 e 110 casos de subnotificação pelo SINAN de escorpionismo e de araneísmo, respectivamente. Esse resultado não era esperado uma vez que estes acidentes são em sua grande maioria classificados como leves $^{8,9}$, não prevendo aplicação de soros, cuja obtenção está condicionada a notificação dos casos à SES-RJ.

Pode-se afirmar que as subnotificações pelo SINAN vêm decrescendo. Entre 2001 e 2005, vinte e três municípios subnotificaram ao SINAN os três tipos de acidente e, em 2005, apenas Resende e Volta Redonda mantiveram esse comportamento.

Os municípios não apresentam uma prática de notificação idêntica para os três tipos de acidentes estudados. O município de Paraíba do Sul subnotificou ao SINAN os acidentes ofídicos e aracnídeos, porém notificou mais casos ao SINAN de acidentes escorpiônicos. O município de Três Rios subnotificou ao SINAN os acidentes ofídicos e escorpiônicos e notificou igualmente às duas bases os acidentes aracnídeos.

\section{Conclusão}

Há um claro aumento da capacidade de captação de dados pelo SINAN ao longo do período. Entretanto, as perdas de registros ainda são grandes, especialmente nos casos de acidentes ofídicos, quando comparados aos casos registrados pela SES-RJ. Isso mostra que a base de dados criada a partir da prática condicionada da troca de fichas por soro, estabelecida na época da criação do Programa de Controle de Acidentes por Animais Peçonhentos e utilizada ainda hoje pela SES-RJ, constitui uma 
sentinela que permite identificar problemas de subnotificação no Estado do Rio de Janeiro. Para as demais unidades da federação é recomendável um estudo semelhante para deliberar a respeito dos procedimentos mais adequados a serem adotados.

Embora o presente estudo tenha se limitado aos municípios e regiões do Estado do Rio de Janeiro, ele pode servir de base para afirmar que é necessário investir esforços no sentido de ampliar a coleta de casos pelo SINAN para garantir a universalização da cobertura da informação sobre esses acidentes não só no Estado do Rio de Janeiro como em todo o território nacional. $\mathrm{O}$ fato de constituir um sistema nacional, integrado aos outros sistemas de informação do DATASUS, e por contar com uma ficha de coleta de dados padronizada, justificam essa preocupação com a melhoria da capacidade de obtenção de dados do sistema.

Deve-se salientar a capacidade do SINAN para gerar relatórios e análises em todos os níveis, com grande potencial de articulação com o nível central e a disponibilização de muitas variáveis de interesse de forma regular e rápida. Outra característica relevante é o fato de o SINAN ser capaz de captar os acidentes ocorridos num Estado, mesmo quando eles são notificados por outro Estado, o que constitui um fator importante para atividades de planejamento do atendimento e prevenção.

A avaliação da base de dados do SINAN e o cálculo de indicadores pelas equipes estaduais, regionais e municipais são atividades imprescindíveis para o bom desempenho das ações relacionadas ao processo de orientação e treinamento para atendimento aos acidentados, verificação da boa distribuição dos pólos de atendimento, bem como para a provisão de quantidades adequadas de soro.

O uso sistemático do SINAN, de forma descentralizada, pode contribuir para a democratização da informação, permitindo que todos os profissionais de saúde tenham acesso à informação e as tornem disponíveis para a comunidade. É, portanto, um instrumento relevante para auxiliar o planejamento da saúde, definir prioridades de intervenção e permitir que seja avaliado o impacto das ações programadas.

Este cenário justifica a recomendação de se elaborar estratégias de busca ativa de casos além da promoção de atividades sensibilizadoras para a importância do envio das notificações para o SINAN.

\section{Referências}

1. Secretaria de Vigilância em Saúde. Departamento de Vigilância Epidemiológica. Sistema de Informação de Agravos de Notificação - Sinan: normas e rotinas. Brasília: Ministério da Saúde; 2006.

2. Bochner, R, Struchiner, CJ. Acidentes por animais peçonhentos e sistemas nacionais de informação. Cad Saúde Pública 2002; 18: 735-46.

3. Carvalho, DM. Grandes sistemas nacionais de informação em saúde: revisão e discussão da situação atual. IESUS 1997; VI(4): 7-46.

4. Luna, EJA. O Sinan e o resgate da informação para a vigilância epidemiológica [Editorial]. Epidemiol Serv Saúde 2004; 13: 133-4.

5. Laguardia, J, Domingues, CMA, Carvalho, C, Lauerman, CR, Macário, E, Glatt, R. Sistema de Informação de Agravos de Notificação (Sinan): desafios no desenvolvimento de um sistema de informação em saúde. Epidemiol Serv Saúde 2004; 13: 135-46.
6. Wen, FH, Cardoso, JLC, Maláque, CMS, França, FOS, Sant'anna, SS, Fernandes, W, Furtado et al. Influência das alterações ambientais na epidemiologia dos acidentes ofídicos e na distribuição geográfica das serpentes de importância médica nos estados de São Paulo e Paraná, 1988-1997. Informe Epidemiológico do SUS 2002; 11: 457.

7. Ministério da Saúde. Secretaria de Vigilância em Saúde. Sistema de Informação de Agravos de Notificação (Sinan). Tabulação de dados - TabNet. Disponível em http://dtr2004.saude.gov.br/sinanweb/ index.php?name=Tnet. [Acessado durante o ano de 2006, para informações de 2001 a 2005].

8. Secretaria de Vigilância em Saúde. Departamento de Vigilância Epidemiológica. Guia de Vigilância Epidemiológica. $6^{a}$ edição. Brasília: Ministério da Saúde; 2005. 
9. Secretaria de Vigilância em Saúde. Manual de Diagnóstico e Tratamento de Acidentes por Animais Peçonhentos. Brasília: Ministério da Saúde; 2001.

10. Coordenação de Vigilância Ambiental em Saúde. Guia básico no atendimento de acidentes por animais peçonhentos. Rio de Janeiro: Secretaria de Estado de Saúde do Rio de Janeiro; 2005.
11. Coordenação de Vigilância Ambiental em Saúde. Acidentes por animais peçonhentos: conhecendo e prevenindo. Rio de Janeiro: Secretaria de Estado de Saúde do Rio de Janeiro; 2005.

Recebido em: 27/08/07 Versão final reapresentada em: 12/11/07 Aprovado em: 04/12/07 